\title{
An Extensive Study of Interstellar Matter in the IC 1396 Region using several Molecular Lines and Transitions of CO and Far-Infrared Maps from IRAS
}

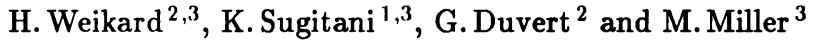 \\ ${ }^{1}$ Department of Astrophysics, Nagoya University, Chikusa-ku, Nagoya 464, Japan \\ ${ }^{2}$ Observatoire de Grenoble, B.P. 53 X, 38041 Grenoble CEDEX, France \\ ${ }^{3}$ I. Physikalisches Institut der Universität zu Köln, Zülpicher Straße 77, 5000 Köln, FRG
}

IC 1396 is an H II region in Cepheus excited by the massive O6.5 star HD 206267. The distance is about $750 \mathrm{pc}$. The region exhibits a number of bright-rimmed molecular clouds in which outflows have been detected (Sugitani et al. 1989, Duvert et al. 1990).

We present preliminary results of a $\mathrm{CO}(1 \rightarrow 0)$ survey of the entire region (Fig. 1) and a multi-line study (Fig. 2 as a first step) based on observations using three small millimeter wave telescopes (see Table 1) which are located at Nagoya, on Plateau de Bure (France) and on Gornergrat (Switzerland). The higher transition observations cover only the bright

\begin{tabular}{|lcccccc|}
\hline Telescope & $\begin{array}{c}\text { Line and } \\
\text { transition }\end{array}$ & $\begin{array}{c}\text { Positions } \\
\text { observed }\end{array}$ & HPBW & Grid & $\begin{array}{c}\text { Resolution } \\
\text { in km/sec }\end{array}$ & $\begin{array}{c}\text { r.m.s.-noise } \\
\text { in K }\end{array}$ \\
\hline Nagoya 4 m & ${ }^{12} \mathrm{CO}(1 \rightarrow 0)$ & 6050 & $2 ! 8$ & $2^{\prime}$ & 0.13 & 1.0 \\
& ${ }^{13} \mathrm{CO}(1 \rightarrow 0)$ & 5000 & $2 ! 9$ & $2^{\prime}$ & 0.08 and 0.135 & 0.25 \\
POM-2 2.5 m & ${ }^{12} \mathrm{CO}(2 \rightarrow 1)$ & 400 & $2 ! 2$ & $2^{\prime}$ & 0.20 & 0.45 \\
& ${ }^{13} \mathrm{CO}(2 \rightarrow 1)$ & 400 & $2 ! 3$ & $2^{\prime}$ & 0.215 & 0.25 \\
KOSMA 3 m & ${ }^{12} \mathrm{CO}(3 \rightarrow 2)$ & 730 & $1^{\prime} ! 5$ & $1^{\prime}$ & 0.145 & 1.3 \\
\hline
\end{tabular}

Table 1 : Observational parameters

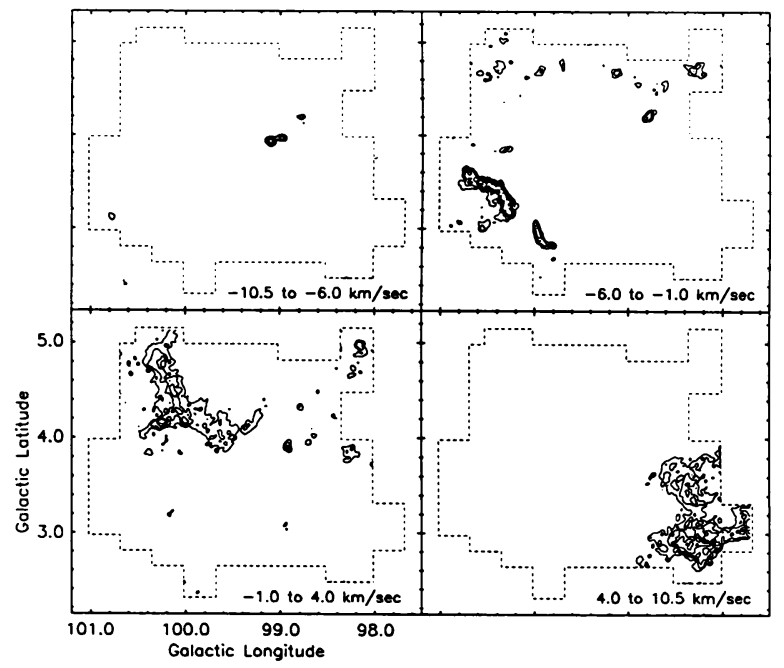

rims $\mathrm{A}, \mathrm{B}$ and $\mathrm{D}$ to $\mathrm{H}$ (designations of Pottasch 1956, see Fig. 2 for some spectra taken on rim E).

Fig. 1 : Distribution and kinematic structure of the molecular material as observed in ${ }^{12} \mathrm{CO}(1 \rightarrow 0)$ at Nagoya

Contour levels are drawn from 8 to $38 \mathrm{~K} \cdot \mathrm{km} / \mathrm{sec}$, the step is $5 \mathrm{~K} \cdot \mathrm{km} / \mathrm{sec}$. The most noticeable features are $\operatorname{rim} \mathrm{G}(-4$ to $-2.5 \mathrm{~km} / \mathrm{sec})$, $\operatorname{rim} B$ $(-5 \mathrm{~km} / \mathrm{sec})$ and the famous rim $A$ $(-8 \mathrm{~km} / \mathrm{sec})$. Rim E $(0.5 \mathrm{~km} / \mathrm{sec})$ suffers from strong self-absorption (cf. Fig.2). The cloud which appear's in the lower right map $(8 \mathrm{~km} / \mathrm{sec})$ is probably in the foreground.

Note the absence of molecular emission in a region of more than one square degree around $(99.4,3.3)$ and compare with Fig. 3! 

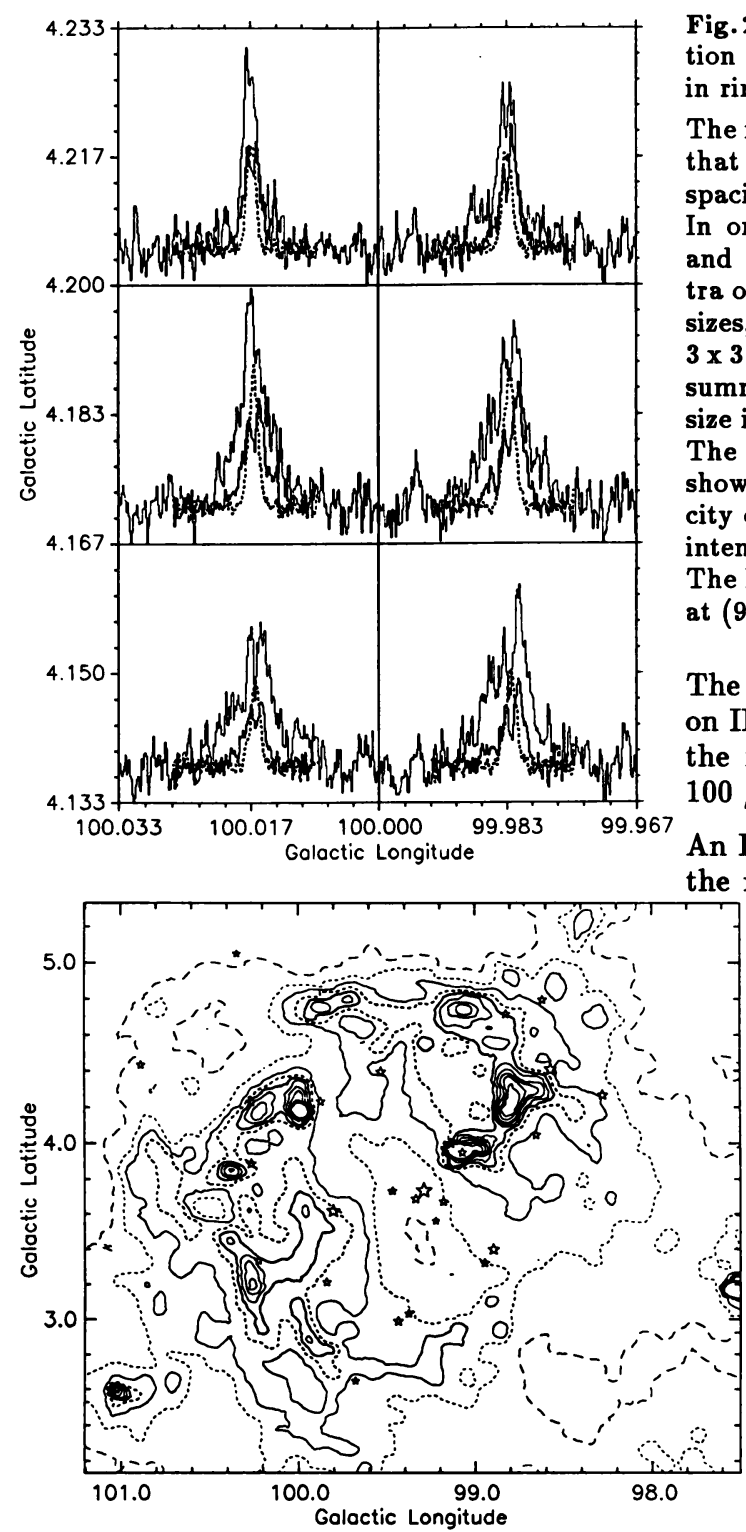

Fig. 2: Spectrum profile map of higher transition lines observed with POM-2 and KOSMA in rim $\mathrm{E}$ where an outflow had been detected The range in LSR velocity is -24 to $24 \mathrm{~km} / \mathrm{sec}$, that in antenna temperature -1 to $6 \mathrm{~K}$. The spacing is $2 ! 0$.

In order to improve the signal-to-noise ratio and to make directly comparable the spectra originally taken with rather different beam sizes, $2 \times 2$ neighbouring $(2 \rightarrow 1)$ spectra and $3 \times 3$ neighbouring $(3 \rightarrow 2)$ spectra have been summed up, respectively. The effective beam size is then about $4 ! 3$.

The ${ }^{12} \mathrm{CO}$ spectra $((2 \rightarrow 1)$ thick, $(3 \rightarrow 2)$ thin $)$ show very strong self-absorption at the velocity of ${ }^{13} \mathrm{CO}(2 \rightarrow 1)$ (dotted). Note the more intensive $(3 \rightarrow 2)$ emission!

The IRAS source responsible for the outflow is at $(99.983,4.170)$.

The bright rims are also very prominent on IRAS Sky Brightness Images in each of the four wavelength bands (cf. Fig. 3 for $100 \mu \mathrm{m})$.

An LVG formalism will be used to analyse the molecular data in order to get fundamental physical parameters like density, temperature and excitation conditions for the bright-rimmed dust clouds.

Besides, we want to apply a multicorrelation analysis to the millimeter and IRAS maps in all four wavelengths disclosing quantitative aspects.

Fig. 3 : $100 \mu \mathrm{m}$ brightness (IRAS coadded map) of the same region as in Fig. 1 Contour levels are drawn from 70 (longdashed) to $250 \mathrm{MJy} / \mathrm{sr}$, the step is $20 \mathrm{MJy} / \mathrm{sr}$.

Stars indicate the positions of $\mathrm{O}$ and B stars: The brightest near the centre is HD 206267.

Acknowledgement: H.W. gratefully acknowledges the financial support from the European Economic Community under contract number ST2-0305.

\section{References}

Duvert, G., J. Cernicharo, R. Bachiller, and J. Gómez-González : Astron. Astrophys. 233, 190 Pottasch, S.: Bull. Astron. Inst. Netherlands 13, 77 (1956)

Sugitani, K., Y.Fukui, A. Mizuno, and N. Ohashi: Astrophys. J. 342, L87 (1989) 